\title{
Achievable Rate of Spectrum Sharing Cognitive Radio Multiple-Antenna Channels
}

\author{
Lokman Sboui, Hakim Ghazzai, Zouheir Rezki, and Mohamed-Slim Alouini \\ Electrical Engineering Program \\ Computer, Electrical and Mathematical Sciences and Engineering (CEMSE) Division \\ King Abdullah University of Science and Technology (KAUST) \\ Thuwal, Makkah Province, Saudi Arabia \\ \{lokman.sboui, hakim.ghazzai, zouheir.rezki, slim.alouini\}@kaust.edu.sa
}

\begin{abstract}
We investigate the spectral efficiency gain of an uplink Cognitive Radio (CR) Multi-Input-Multi-Output system in which the Secondary User (SU) is allowed to share the spectrum with the Primary User (PU) using a specific precoding scheme to communicate with a common receiver. The proposed scheme exploits, at the same time, the free eigenmodes of the primary channel after a space alignment procedure and the interference threshold tolerated by the PU. At the common receiver, we adopt a Successive Interference Cancellation (SIC) technique to eliminate the effect of the detected primary signal transmitted through the exploited eigenmodes. Furthermore, we analyze the SIC operation inaccuracy as well as the CSI estimation imperfection on the PU and SU throughputs. Numerical results show that our proposed scheme enhances considerably the cognitive achievable rate. For instance, in case of a perfect detection of the PU signal, the CR rate remains non-zero for high Signal to Noise Ratio which is usually impossible when we only employ a space alignment technique. We show that a modified water-filling power allocation policy at the PU can increase the secondary rate with a marginal degradation of the primary rate. Finally, we investigate the behavior of the $P U$ and $S U$ rates through the study of the rate achievable region.
\end{abstract}

Index Terms-Achievable rate, underlay cognitive radio, spectrum sharing, space alignment, successive interference cancellation, rate region.

\section{INTRODUCTION}

Due to the spread of the current wireless services and wireless communication evolution, more bandwidth is needed to offer more high data rate services. Consequently, the accessible radio spectrum is becoming critically scarce as describes the Federal Communications Commission (FCC) [1]. To overcome this shortage, current spectrum allocation policy, relatively inefficient, should be substituted by an optimized spectrum management concept that avoids unused spectrum holes. In this vision, the Cognitive Radio (CR) concept was introduced by Mitola [2] in order to optimize the use of the spectrum within multiple users. The main idea is to allow secondary (non-licensed/cognitive) users, noted "SU", to share the spectrum with the primary (licensed/non cognitive) users, noted "PU", without affecting the primary communication.

\footnotetext{
* Part of this work has been presented at the 2013 International Conference on Communications (ICC'13), Budapest, Hungary.

* The work of M. -S. Alouini and Z. Rezki was supported by the Qatar National Research Fund (a member of Qatar Foundation) under NPRP Grant NPRP 5-250-2-087. The statements made herein are solely the responsibility of the authors
}

During last years, three main CR techniques where introduced [3]: underlay, overlay, and interweave. Many studies were focusing on determining the performances of the SU when such techniques are adopted. To this end, one has to distinguish two CR strategies:

- Orthogonal transmission: in which the PU does not "feel" the SU communication at all. The SU spots spectrum holes in (space, time and frequency) then opportunistically performs communication. This strategy is called: interweave CR mode [4].

- Non-orthogonal transmission: in which the SU is allowed to communicate simultaneously with the PU. However, the secondary communication is limited to a certain interference-temperature tolerated by the primary user [5], [6]. This strategy is called underlay CR mode.

The interweave mode is based on the strategy of communicating in the non-used primary resources [7]. Thus, there is no direct effect on the primary communication performances. Meanwhile, this requires a continuous channel sensing from the SU in order to detect the activity/inactivity of the PU [8]. However, the resulting SU rate is limited and can be zero in the case of excessive or full PU activity. On the other hand, in the underlay mode, the PU is willing to tolerate some interference caused by the SU by defining a certain predefined threshold to be respected [3]. This mode allows the SU to transmit even if the PU is at full activity. Furthermore, the adoption of the Multiple-Input and Multiple-Output (MIMO) antenna in CR [9], was shown to be beneficial to the SU as well as the PU [10].

In previous works [11]-[13], the authors adopted a specific precoding/decoding technique to avoid interference between the PU and the SU. In particular, a space alignment technique was adopted in MIMO CR scenarios. This technique is based on a precoding that aligns the PU eigenmodes in the MIMO channel resulting in some unused eigenmodes that are exploited by the SU. However, in this setting the SU rate can be zero when the PU is in a high power regime [14]. In [15], a modified water-filling power allocation [16] was adopted to ensure a positive SU rate. However, the PU rate, in this case, suffers a rate degradation.

In this paper, we investigate the combination of both orthogonal and non-orthogonal CR transmission modes in an uplink MIMO communication. Our objective is to examine 
the maximum achievable rate (expressed in bits per channel use "BPCU") for the SU over all channel realizations. The common destination scenario was widely studied in [17]-[21]. In this scenario, the common base station is able to accept opportunistic transmissions from an eventual SU who wants to share the PU spectrum. This strategy can be adopted by cellular service provider to satisfy the need of two types of users: (i) licensed users that benefit from a reliable Quality of Service (QoS) and have to pay the ordinary service cost, (ii) non-licensed users that share the PU spectrum to gain some rate (possibly at a lower QoS) but with lower prices. Another application of the spectrum sharing with common receiver is the recent Long Term Evolution Unlicensed (LTE-U) technique where users can share an unlicensed spectrum through a common access point or base station [22]. In addition, we present a new precoding/decoding scheme that exploits the PU tolerated interference, allowing the SU to achieve higher rate with minimal effect on the PU communication. At the common receiver, the PU signal transmitted through the exploited eigenmodes is decoded first as it is expected to have the higher signal (the SU signal is always limited by the interference threshold forced by the PU). Once the effect of the PU signal is eliminated from the received signal using a Successive Interference Cancellation (SIC) decoder [23], the SU signal is decoded. In addition, we study the SU rate depending on the PU signal detection accuracy. Moreover, we investigate the impact of a modified power allocation policy on the SU rate depending on the PU tolerance. This policy presents a certain trade-off between the PU and SU rates that may be applied within pricing rewards in hybrid networks, i.e., including cognitive and non-cognitive users. Finally, we study the rate region to fully describe the contributions of this work. The proposed scheme can be employed in a practical cellular communication scenario in which primary and secondary mobile stations (MS) tend to communicate with the same base station (BS) using the same frequency. The primary MS and the BS belong to the same operator whereas the secondary MS belongs to another operator that has no coverage in the current location. The secondary MS can use the primary infrastructure in a roaming mode that is governed by an agreement pricing policy between service providers.

Our contribution, in this paper, are as follows:

- We adopt a space alignment technique along with an interference temperature threshold technique to ensure a non-zero SU rate.

- We study the effect of the imperfection of both, the SIC at the base station and the CSI estimation at the transmitters, on the SU power allocation.

- We investigate the impact of a tolerated rate degradation from the PU on the SU performance.

- We analyze the proposed scheme PU-SU rate region.

The rest of this paper is organized as follows. In Section II, the system model is presented. Section III describes the precoding and decoding strategies and derive the SU achievable rate. The modified water-filling method leading to optimized secondary rate with PU tolerance is described in Section IV. The rate region study is presented in Section V. Finally, the

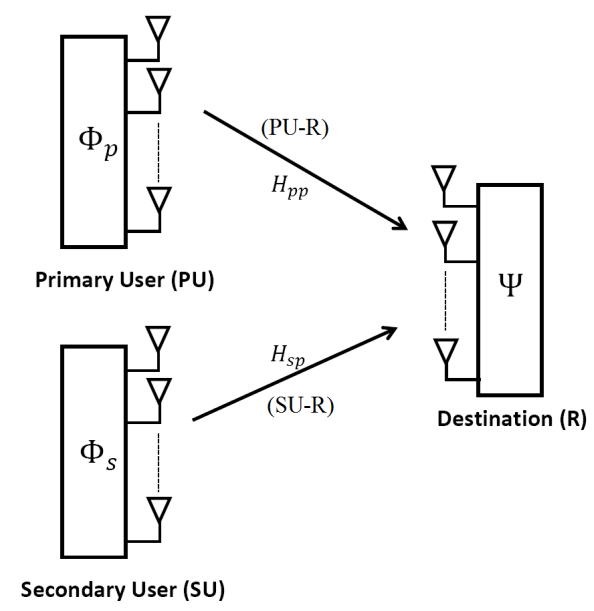

Figure 1: Uplink cognitive radio system with $N$ antennas.

paper is concluded in Section VI.

\section{SySTEM MODEL}

We consider an uplink communication system consisting of two transmitters "PU" and "SU" and a common receiver noted "R". The PU, as a licensed user, exploits the channel while the SU, as an unlicensed node, is allowed to share opportunistically the spectrum and to access the channel under some constraints to maintain a certain QoS of the primary communication. We assume that each node is equipped with $N$ antennas, and the channel gain matrices representing the links between the PU and R (PU-R) and between SU and $\mathrm{R}$ (SU-R) are denoted by $\boldsymbol{H}_{p p}$ and $\boldsymbol{H}_{\boldsymbol{s p}}$, respectively, as shown in Fig.1. In the framework of an uplink scenario where the receiver is common to both transmitters, the interference may cause a significant deterioration to both primary and secondary performances. However, by adopting an interference temperature protection [24], the PU tolerates an interference level per receive antenna denoted $I_{\text {peak }}$. That is, if the SU satisfies this constraint, we consider that the QoS of the PU is not affected (i.e., the PU is able to achieve its required rate). This threshold is actually controlled by the receiver that knows the power levels and channel conditions of the primary link and decides which is the maximum threshold that keeps the PU satisfied where maximizing the eventual SU rate gain. On the other hand, we exploit the interference alignment technique presented in [11] to enable the SU to access the channel without causing any interference to the PU by applying a simple linear unitary precoding, described in Section III. We assume that full Channel State Information (CSI) is available at the receiver and the secondary transmitter (i.e., PU-R and SU$\mathrm{R}$ channel gains). However, the primary transmitter is assumed to only know the PU-R CSI. The received signal $y$ at the receiver, $R$, is expressed as follows

$$
y=H_{p p} \Phi_{p} s_{p}+H_{s p} \Phi_{s} s_{s}+w,
$$

where $\boldsymbol{H}_{p p}$ and $\boldsymbol{H}_{s p}$ are assumed to be independent, $\boldsymbol{\Phi}_{p}$ and $\Phi_{s}$ are the linear precoding matrices applied at the PU and SU, respectively. The signal transmitted by the PU and SU are 
denoted $s_{p}$ and $s_{s}$, respectively. For $i \in\{p, s\}$, we consider $\boldsymbol{P}_{\boldsymbol{i}}=\mathbb{E}\left[\boldsymbol{s}_{\boldsymbol{i}} \boldsymbol{s}_{\boldsymbol{i}}{ }^{h}\right]$ to be the covariance matrix of the vector $s_{i}$, where $\mathbb{E}[\cdot]$ is the expectation operator and ${ }^{h}$ designates the transpose conjugate operator. This covariance matrix is subject to a power constraint $\operatorname{Tr}\left(\boldsymbol{\Phi}_{\boldsymbol{i}} \boldsymbol{P}_{\boldsymbol{i}} \boldsymbol{\Phi}_{\boldsymbol{i}}^{\boldsymbol{h}}\right) \leq P_{t o t, i}$ where $\operatorname{Tr}(\boldsymbol{A})=\sum_{j} A(j, j)$ is the trace of the matrix $\boldsymbol{A}$, and $P_{t o t, i}$ is the total power budget of user $i$. Finally, $\boldsymbol{w}$ indicates a zero mean additive white Gaussian noise (AWGN) vector at the receiver with an identity covariance matrix; $\boldsymbol{I}_{\boldsymbol{N}}$.

\section{Precoding and Decoding Strategy}

In this section, we present the proposed linear precoding and decoding techniques in order to maximize the rate of the SU without degrading the PU QoS by respecting an interference constraint. Meanwhile, we introduce the interference alignment technique, presented in [11], which permits to the SU to transmit through the unused primary eigenmodes.

\section{A. Problem Formulation}

By having a perfect CSI of the PU-R link at the PU transmitter, the PU can optimally allocate its power to maximize the achievable rate using a water-filling policy. By applying a Singular Value Decomposition (SVD) to $\boldsymbol{H}_{p \boldsymbol{p}}$, the PU transmits through parallel channels characterized by the associated eigenmodes. The SVD of the matrix is denoted $\boldsymbol{H}_{p p}=\boldsymbol{U} \boldsymbol{\Lambda} \boldsymbol{V}^{h}$ where $\boldsymbol{U}$ and $\boldsymbol{V}$ are two unitary matrices and $\boldsymbol{\Lambda}$ is a diagonal matrix that contains the ordered singular values of $\boldsymbol{H}_{p p}$ denoted as $\lambda_{1} \geq \lambda_{2} \geq \cdots \geq \lambda_{N}$. Note that the eigenvalues of $\boldsymbol{H}_{p p}$ are equivalent to the squares of its singular values since it is a square matrix. To transform the MIMO channel to $N$ parallel channels, we perform linear precoding and decoding at the transmitter and at the receiver, respectively. Let

$$
\Phi_{p}=\boldsymbol{V} \text { and } \boldsymbol{\Psi}=\boldsymbol{U}
$$

then, the output received signal after decoding becomes:

$$
\boldsymbol{r}=\boldsymbol{\Psi}^{h} \boldsymbol{y}=\boldsymbol{\Lambda} \boldsymbol{s}_{\boldsymbol{p}}+\boldsymbol{U}^{h} \boldsymbol{H}_{\boldsymbol{s p}} \boldsymbol{\Phi}_{\boldsymbol{s}} \boldsymbol{s}_{\boldsymbol{s}}+\tilde{\boldsymbol{w}},
$$

where $\tilde{\boldsymbol{w}}=\boldsymbol{\Psi}^{h} \boldsymbol{w}$ remains a zero mean AWGN with a covariance matrix $\boldsymbol{I}_{N}$, since $\Psi$ is unitary. Being a licensed user, the PU ignores the existence of SU, takes into account an eventual interference that does not exceed a fixed $I_{\text {peak }}$ (i.e., $\boldsymbol{S} \boldsymbol{S}^{h} \leq I_{\text {peak }}$ where $\boldsymbol{S}=\boldsymbol{U}^{h} \boldsymbol{H}_{\boldsymbol{s} p} \boldsymbol{\Phi}_{s} \boldsymbol{s}_{\boldsymbol{s}}$ ). In fact, $I_{\text {peak }}$ is a parameter controlled by the common receiver to permit the PU to achieve its required throughput and allow the SU to communicate simultaneously. The PU applies a water-filling Power Allocation (WPA) [11] to maximize its achievable rate $R_{p}$ by solving the following optimization problem

$$
\begin{aligned}
& \max _{\boldsymbol{P}_{\boldsymbol{p}}} R_{p}=\log _{2}\left(\operatorname{det}\left(\boldsymbol{I}_{\boldsymbol{N}}+\frac{\boldsymbol{P}_{\boldsymbol{p}} \boldsymbol{\Lambda} \mathbf{\Lambda}^{h}}{I_{\text {peak }}+1}\right)\right) \\
& \text { subject to } \operatorname{Tr}\left(\boldsymbol{P}_{\boldsymbol{p}}\right) \leq P_{\text {tot }, p .} .
\end{aligned}
$$

The solution of this WPA problem is given in [25] by:

$$
P_{p}(j, j)=\left[\frac{1}{\mu_{0}}-\frac{1+I_{\text {peak }}}{\lambda_{j}^{2}}\right]^{+}, \forall j=1, \ldots, N
$$

where $[.]^{+}=\max (0,$.$) and \mu_{0}$ is the Lagrangian multiplier corresponding to the primary total power constraint.

Note that in some cases, when the channel gain is poor, the number of used eigenmodes by the PU can be less than $N$ when $\frac{1}{\mu_{0}}-\frac{1+I_{\text {peak }}}{\lambda_{j}{ }^{2}} \leq 0$ for some $j^{\prime} s$. Consequently, the unused eigenmodes could be freely exploited by the SU. Let $n(0 \leq$ $n<N$ ) be the number of unused eigenmodes, we distinguish two sets of eigenmodes: $N-n$ eigenmodes used by the PU and $n$ unused eigenmodes. In order to totally eliminate the effect of interference, an appropriate choice of $\boldsymbol{\Phi}_{s}$ has been proposed in [11] as $\left(\boldsymbol{H}_{\boldsymbol{s p}}\right)^{-1} \boldsymbol{U} \overline{\boldsymbol{P}}_{\boldsymbol{p}}$, where $\overline{\boldsymbol{P}}_{\boldsymbol{p}}$ is a diagonal matrix with the following entries:

$$
\bar{P}_{p}(j, j)=\left\{\begin{array}{ll}
1 & \text { if } P_{p}(j, j)=0 \\
0 & \text { if } P_{p}(j, j) \neq 0,
\end{array} \forall j=1, \ldots, N .\right.
$$

This choice of $\boldsymbol{\Phi}_{s}$ prevents the SU to share the used eigenmodes and force it to only use the free ones. In our framework, however, we allow the SU to transmit in all the eigenmodes by respecting a certain interference temperature threshold $I_{\text {peak }}$ when sharing the used eigenmodes. Hence, $\boldsymbol{\Phi}_{s}$ should satisfy $\boldsymbol{U}^{h} \boldsymbol{H}_{\boldsymbol{s} p} \boldsymbol{\Phi}_{\boldsymbol{s}}=\boldsymbol{I}_{\boldsymbol{N}}$. Consequently, we choose $\boldsymbol{\Phi}_{\boldsymbol{s}}$ as follows:

$$
\boldsymbol{\Phi}_{\boldsymbol{s}}=\left(\boldsymbol{H}_{\boldsymbol{s p}}\right)^{-1} \boldsymbol{U} .
$$

Note that when all PU eigenmodes are free, the SU can freely transmit without being subject to the PU interference constraint. In this case, the SU applies a WPA to maximize its achievable rate without requiring any particular precoding scheme. However, in order for the proposed precoding to be implemented, the SU needs to know in addition to its own CSI $H_{s p}$, the unitary matrix $U$ as well. This assumption is realistic to a certain extent as feedback CSI is adopted in most wireless communication protocols. Furthermore, adopting such an assumption allows to capture an upper bound of the corresponding performance. Consequently, the output received signal becomes:

$\boldsymbol{r}=\left[\begin{array}{cc}\tilde{\boldsymbol{\Lambda}} & 0 \\ 0 & 0\end{array}\right]_{N \times N}\left[\begin{array}{c}\boldsymbol{s}_{\boldsymbol{p}^{(1)}} \\ 0\end{array}\right]_{N \times 1}+\left[\begin{array}{c}\boldsymbol{s}_{\boldsymbol{s}}^{(1)} \\ \boldsymbol{s}_{\boldsymbol{s}}^{(2)}\end{array}\right]_{N \times 1}+\left[\begin{array}{c}\tilde{\boldsymbol{w}}^{(1)} \\ \tilde{\boldsymbol{w}}^{(2)}\end{array}\right]_{N \times 1}$

where $\tilde{\boldsymbol{\Lambda}}=\operatorname{diag}\left[\lambda_{1} \cdots \lambda_{N-n}\right]$ (i.e., the $N-n$ non-zeros eigenvalues of $\boldsymbol{\Lambda}), \boldsymbol{s}_{\boldsymbol{p}}{ }^{(1)}$ is a vector composed by the $N-n$ transmitted PU signal through the $N-n$ first antennas, $\boldsymbol{s}_{\boldsymbol{s}}{ }^{(1)}$ and $\boldsymbol{s}_{\boldsymbol{s}}{ }^{(2)}$ correspond to the vectors composed by the transmitted SU signal through the $N-n$ used and $n$ unused eigenmodes, respectively. The received signal is expressed as

$$
\begin{aligned}
& r_{j}=\lambda_{j} s_{p_{j}}+s_{s j}+\tilde{w}_{j}, \forall j=1, \cdots, N-n, \\
& r_{j}=s_{s j}+\tilde{w}_{j}, \forall j=N-n+1, \cdots, n .
\end{aligned}
$$

The decoding of the SU signal is performed after applying the SIC to estimate the strongest signal: $s_{p}$. Typically, the SU signal is always limited by the interference threshold forced by the PU [26]. Note that it is natural to consider that $s_{p}$, the signal from a licensed user, is stronger than the constrained SU signal. Note also that the SU signal transmitted over the $n$ free eigenmodes is only constrained by a total power constraint. Next, we analyze the achievable rate of SU using the proposed technique depending on the perfectness 
of the SIC operation. We, first, analyze the performance of the system when a fully successful SIC is applied. Then, we investigate the gain in performance with an imperfect SIC (i.e., fully erroneous). We introduce, afterwards, a realistic scenario in which the SIC is partially successful by including a parameter $\alpha(0 \leq \alpha \leq 1)$ that corresponds to the probability of detecting the PU signal $s_{p}$ correctly before applying the SIC. The success of this step is contingent on the Signal to Interference plus Noise Ration (SINR) of the transmitted signal, i.e., $\alpha=\operatorname{Prob}\left\{S I N R \geq S I N R_{t h}\right\}$, where $S I N R_{t h}$ denotes the pre-defined threshold that identifies whether a communication is successful or not [26].

\section{B. Perfect SIC}

We consider that the PU message is always decoded perfectly, i.e. $\hat{s_{p_{j}}}=s_{p_{j}}, \forall j=1, \cdots, N-n$, where $\hat{s_{p_{j}}}$ is the estimated PU signal at the $\mathrm{j}^{\text {th }}$ receiving antenna. Hence, the cancellation of the PU effect is performed correctly $(\alpha=1)$ and, in this case, the output received signal after the SIC decoding, $\tilde{\boldsymbol{r}}$, is written as

$$
\tilde{\boldsymbol{r}}=\boldsymbol{r}-\Lambda \hat{s}_{p}=\boldsymbol{s}_{s}+\tilde{\boldsymbol{w}} .
$$

Note that, the secondary channel becomes unitary due to the specific precoding described in (8) which provides a throughput that is independent of the secondary channel gain. Consequently, the corresponding achievable rate $R_{s}{ }^{(\alpha=1)}$ is given by

$$
\max _{\boldsymbol{P}_{\boldsymbol{s}}} R_{s}{ }^{(1)}=\sum_{j=1}^{N} \log _{2}\left(1+P_{s}(j, j)\right) .
$$

Thus, to find the optimal power allocation $\boldsymbol{P}_{s}^{*}$, we have to solve the following optimization problem:

$$
\begin{aligned}
\max _{\boldsymbol{P}_{\boldsymbol{s}}} & R_{s}{ }^{(1)}=\sum_{j=1}^{N} \log _{2}\left(1+P_{s}(j, j)\right) \\
& \text { subject to } \operatorname{Tr}\left(\boldsymbol{\Phi}_{\boldsymbol{s}} \boldsymbol{P}_{\boldsymbol{s}} \boldsymbol{\Phi}_{\boldsymbol{s}}{ }^{h}\right) \leq P_{t o t, s} \\
& \text { and } P_{s}(j, j) \leq I_{\text {peak }}, \forall j=1, \cdots, N-n .
\end{aligned}
$$

By using the invariance of the Trace operator under the cyclic permutation, the constraint (14) could by written as:

$$
\operatorname{Tr}\left(\boldsymbol{\Phi}_{\boldsymbol{s}}{ }^{h} \boldsymbol{\Phi}_{\boldsymbol{s}} \boldsymbol{P}_{\boldsymbol{s}}\right) \leq P_{t o t, s} .
$$

Let the matrix $\boldsymbol{A}_{s}=\boldsymbol{\Phi}_{s}{ }^{h} \boldsymbol{\Phi}_{s}$, the constraint (14) becomes:

$$
\operatorname{Tr}\left(\boldsymbol{A}_{\boldsymbol{s}} \boldsymbol{P}_{\boldsymbol{s}}\right) \leq P_{t o t, s} \text {. }
$$

Since the objective function is convex and the constraints are linear, we use the Lagrange method [27]. The resulting power profile is formulated in the following theorem.

\section{Theorem}

The solution to the optimization problem defined by (13) is

$$
\begin{aligned}
& P_{s}^{*}(j, j)= \\
& \left\{\begin{array}{c}
\min \left\{\left[\frac{1}{\mu A_{s}(j, j)}-1\right]^{+}, I_{\text {peak }}\right\}, \forall j=1, \cdots, N-n, \\
{\left[\frac{1}{\mu A_{s}(j, j)}-1\right]^{+}, \forall j=N-n+1, \cdots, N,}
\end{array}\right.
\end{aligned}
$$

where $\mu$ is the Lagrange multiplier associated to the average power constraint.

Proof: The proof is presented in the Appendix.

Note that, the optimal power does not depend on the primary transmission but only on $I_{p e a k}$ and $P_{t o t, s}$ through $\mu$.

\section{Imperfect SIC}

In Subsection III-B, we considered the ideal case when capacity achieving codes are employed by the PU transmitter. Since the PU rate is smaller than the PU mutual information, arbitrary low decoding error probability is achievable. In this subsection, we assume that instead of using capacity achieving codes, PU employs more practical coding schemes and thus decoding errors are unavoidable no matter how small the PU rate is. In this case, we investigate the extreme scenario $(\alpha=0)$ when the receiver decodes the cognitive message after employing an imperfect SIC where the interference power at each antenna is equal to $\mathbb{E}\left[\left|\tilde{\lambda}_{j}\left(s_{p_{j}}-\hat{s}_{p_{j}}\right)\right|^{2}\right]=2 P_{p}(j, j) \lambda_{j}{ }^{2}$. Then, the SU rate is obtained by solving the following optimization problem:

$$
\begin{aligned}
& \max _{\boldsymbol{P}_{\boldsymbol{s}}} R_{s}{ }^{(0)}=\sum_{j=1}^{N-n} \log _{2}\left(1+\frac{P_{s}(j, j)}{1+2 P_{p}(j, j) \lambda_{j}{ }^{2}}\right) \\
& +\sum_{j=N-n+1}^{N} \log _{2}\left(1+P_{s}(j, j)\right) \\
& \text { subject to } \operatorname{Tr}\left(\boldsymbol{A}_{\boldsymbol{s}} \boldsymbol{P}_{\boldsymbol{s}}\right) \leq P_{\text {tot }, s} \\
& \text { and } P_{s}(j, j) \leq I_{\text {peak }}, \forall j=1, \cdots, N-n .
\end{aligned}
$$

In this scenario, the optimal power is computed similarly to the perfect SIC case by using the Lagrange method, the optimal power is given by

$P_{s}^{*}(j, j)=\left\{\begin{array}{c}\min \left\{\left[\frac{1}{\mu A_{s}(j, j)}-\left(1+2 P_{p}(j, j) \lambda_{j}^{2}\right)\right]^{+}, I_{\text {peak }}\right\} \\ \forall j=1, \cdots, N-n, \\ {\left[\frac{1}{\mu A_{s}(j, j)}-1\right]^{+}, \forall j=N-n+1, \cdots, N,}\end{array}\right.$

where $\mu$ is the Lagrange multiplier associated to the total power constraint. We notice, here, that the optimal power depends on the primary power and eigenmodes which means that the secondary is adapting its power continuously with the variation of the primary channel state.

\section{Erroneous SIC}

We suppose, in this case, that the SIC is performed with an error probability of $1-\alpha$ and then the output received signal, depending on the value of $\alpha$, is given by:

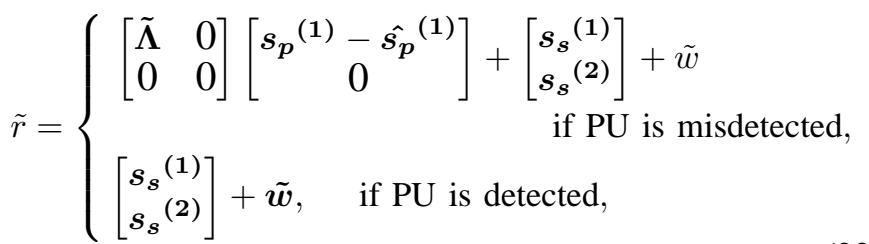


where ${\hat{s_{p}}}^{(1)}$ is a wrong estimate of $s_{p}{ }^{(1)}$. In this case, the optimization problem to maximize the achievable rate $R_{s}^{(\alpha)}$ can be written as follows:

$$
\begin{aligned}
& \max _{\boldsymbol{P}_{\boldsymbol{s}}} R_{s}{ }^{(\alpha)}=(1-\alpha) \sum_{j=1}^{N-n} \log _{2}\left(1+\frac{P_{s}(j, j)}{1+2 P_{p}(j, j) \lambda_{j}{ }^{2}}\right) \\
& +\alpha \sum_{j=1}^{N-n} \log _{2}\left(1+P_{s}(j, j)\right)+\sum_{j=N-n+1}^{N} \log _{2}\left(1+P_{s}(j, j)\right) \\
& \text { subject to } \operatorname{Tr}\left(\boldsymbol{A}_{\boldsymbol{s}} \boldsymbol{P}_{\boldsymbol{s}}\right) \leq P_{\text {tot }, s} \\
& \text { and } P_{s}(j, j) \leq I_{\text {peak }}, \forall j=1, \cdots, N-n .
\end{aligned}
$$

The corresponding optimal power is computed similarly to previous cases using the Lagrange method. Due to the introduction of $\alpha$, the optimal power profile becomes

$$
\begin{aligned}
& P_{s}^{*}(j, j)= \\
& \left\{\begin{array}{c}
\min \left\{\left[f\left(\mu, A_{s}(j, j), \alpha, P_{p}(j, j), \lambda_{j}\right)-1\right]^{+}, I_{\text {peak }}\right\} \\
\forall j=1, \cdots, N-n, \\
{\left[\frac{1}{\mu A_{s}(j, j)}-1\right]^{+}, \forall j=N-n+1, \cdots, N,}
\end{array}\right.
\end{aligned}
$$

where the function $f$ is obtained after solving a second degree equation and is given, for $j=1, \cdots, N-n$, by (25) in the next page. We notice that the expression of the optimal power in (24) is a general expression that involves the cases of perfect and imperfect SIC for $\alpha=1$ and $\alpha=0$, respectively. For instance, if $\alpha=1$ the power profile reduces to (18) while for $\alpha=0$ the power profile becomes (21).

\section{E. Imperfect CSI}

The case of perfect CSI presents an upper bound of the SU performances. However, this assumption is not always true for real systems. Therefore, we present an analysis of the system performances when the CSI is imperfect for both links, i.e., PU-R and SU-R. Each transmitter performs an instantaneous estimation of the channel gain matrix. This estimation adopts a minimum mean square error (MMSE) scheme and the resulting estimated matrices can be presented as

$$
\boldsymbol{H}_{i}=\hat{\boldsymbol{H}}_{i}+\tilde{\boldsymbol{H}}_{i} \quad i=\{p p, s p\}
$$

where $\hat{\boldsymbol{H}}_{i}$ is estimated channel gain matrix and $\tilde{\boldsymbol{H}}_{i}$ is its respective estimation error, $i=\{p p, s p\}$. These errors are independent of each other and have i.i.d. zero-mean complex Gaussian entries with variances $\sigma_{p p}^{2}$ and $\sigma_{s p}^{2}$, respectively. Meanwhile, we denote by $\hat{\boldsymbol{H}}_{p s}$ the channel gain matrix of the PU-R link estimated at the SU with error variance $\sigma_{p s}^{2}$. For simplicity, we assume that the estimation error at the SU transmitter is the same and equal to $\sigma_{s}^{2}$, i.e., $\sigma_{s p}^{2}=\sigma_{p s}^{2}=\sigma_{s}^{2}$.

From one side, the PU performs the SVD, i.e. $\hat{\boldsymbol{H}}_{p p}=$ $\hat{\boldsymbol{U}} \hat{\boldsymbol{\Lambda}} \hat{\boldsymbol{V}}^{h}$, and chooses its precoding and decoding matrices as follows

$$
\hat{\boldsymbol{\Phi}}_{p}=\hat{\boldsymbol{V}} \text { and } \hat{\boldsymbol{\Psi}}=\hat{\boldsymbol{U}} \text {. }
$$

Then, the PU performs the WFA which results in $\hat{n}_{p p}$ free eigenmodes. From the other side, the SU performs the SVD of $\hat{\boldsymbol{H}}_{p s}$ and determine the number of free eigenmodes $\hat{n}_{p s}$ and then chooses its precoding matrix $\hat{\boldsymbol{\Phi}}_{s}=\left(\hat{\boldsymbol{H}}_{p s}\right)^{-1} \hat{\boldsymbol{U}}$. Similarly to (18), we set $\hat{\boldsymbol{A}}_{s}=\hat{\boldsymbol{\Phi}}_{s}^{h} \hat{\boldsymbol{\Phi}}_{s}$, and the corresponding optimal $\mathrm{SU}$ power is given by

$$
\begin{aligned}
& P_{s}^{*}(j, j)= \\
& \left\{\begin{array}{c}
\min \left\{\left[\frac{1}{\mu \hat{A}_{s}(j, j)}-1\right]^{+}, I_{\text {peak }}\right\}, \forall j=1, \cdots, N-\hat{n}_{p s}, \\
{\left[\frac{1}{\mu \hat{A}_{s}(j, j)}-1\right]^{+}, \forall j=N-\hat{n}_{p s}+1, \cdots, N,}
\end{array}\right.
\end{aligned}
$$

Note that there are two levels of channel imperfection: i) imperfection caused by the channel estimation error at both transmitters $\left(\boldsymbol{H}_{p p} \neq \hat{\boldsymbol{H}}_{p p}\right.$ and $\left.\boldsymbol{H}_{s p} \neq \hat{\boldsymbol{H}}_{s p}\right)$ ii) imperfection caused by the mis-alignment of the number of free eigenmodes at both the PU and the $\mathrm{SU}\left(\hat{n}_{p s} \neq \hat{n}_{p p}\right)$ which adds a further degradation of the two performances. In the numerical results, we quantify this degradation to highlight the effect on the system rates.

\section{F. Numerical Results}

We adopt a Rayleigh fading channel in which the entries of $\boldsymbol{H}_{p p}$ and $\boldsymbol{H}_{s p}$ are complex Gaussian random variables with zero mean and unit variance. In Fig. 2.a, we plot the SU achievable rate expressed in (12) as a function of $P_{t o t}=P_{t o t, p}=P_{t o t, s}$ for $N=4$ antennas when the SIC is perfectly performed. The Free Eigenmodes (FE) and Interference Eigenmodes (IE) precoding rates are compared to a precoding with only Free Eigenmodes power allocation (FE only). In Fig. 2.a we show that the interference tolerance allows the SU to achieve better rates when $P_{t o t}$ is greater than $5 \mathrm{~dB}$. In addition, as $P_{t o t}$ increases, the achievable rate reaches a maximum before decreasing to lower asymptotic values, for fixed $I_{\text {peak }}$. This variation in the secondary rate is related to the variation of the number of the free eigenmodes $n$. In fact at low $P_{\text {tot }}$ values, more free eigenmodes are available, however due to the low available power the SU is not able to exploit them and it is optimal to spend the full available power on the strongest eigenmode. This mimics somehow communication over MIMO channels at low power regime, e.g., [28]. Whereas, at high $P_{t o t}$ values, the primary is using all the available eigenmodes and the secondary is only aggregating on these modes while respecting the interference constraint. Consequently, the secondary rate reaches a maximum in the mid-range values of $P_{t o t}$. Thus, the secondary transmitter should be aware about the optimal power $P_{t o t}$ that achieves the maximum rate otherwise the rate will decrease as $P_{t o t}$ exceeds this optimal power shown in Fig. 2.a. At high values of $P_{t o t}, \mathrm{PU}$ is using all its eigenmodes. Then, the SU is transmitting while respecting $I_{\text {peak }}$ over all its antennas. Thus, its rate converges to $N \log _{2}\left(1+I_{\text {peak }}\right)$. In this case, when $I_{\text {peak }} \rightarrow+\infty$, the SU is transmitting freely and its rate converges to the $\mathrm{SU}$ with no $\mathrm{PU}$ case (i.e., red curve in Fig. 2.a). When $I_{\text {peak }} \rightarrow 0$, the SU rate goes to 0 as there is no free eigenmodes. This figure demonstrates also the validity of the derived analytical solution expressed in (18) which is compared to the optimal solution obtained by simulations. In Fig. 2.b, we consider an imperfect SIC scheme 


$$
\begin{aligned}
& f\left(\mu, A_{s}(j, j), \alpha, P_{p}(j, j), \lambda_{j}\right)= \\
& \frac{1}{2 \mu A_{s}(j, j)}\left[1-2 \mu A_{s}(j, j) P_{p}(j, j) \lambda_{j}{ }^{2} \sqrt{1+4(2 \alpha-1) \mu A_{s}(j, j) P_{p}(j, j) \lambda_{j}{ }^{2}+\left(2 \mu A_{s}(j, j) P_{p}(j, j) \lambda_{j}{ }^{2}\right)^{2}}\right] .
\end{aligned}
$$

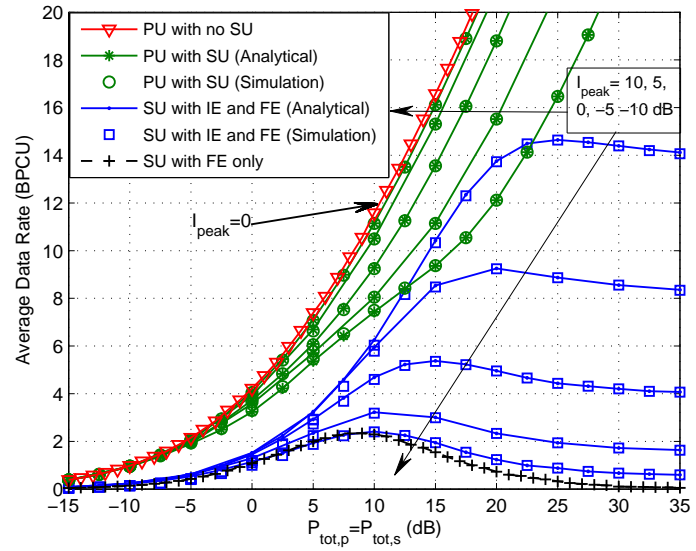

(a) Perfect SIC.

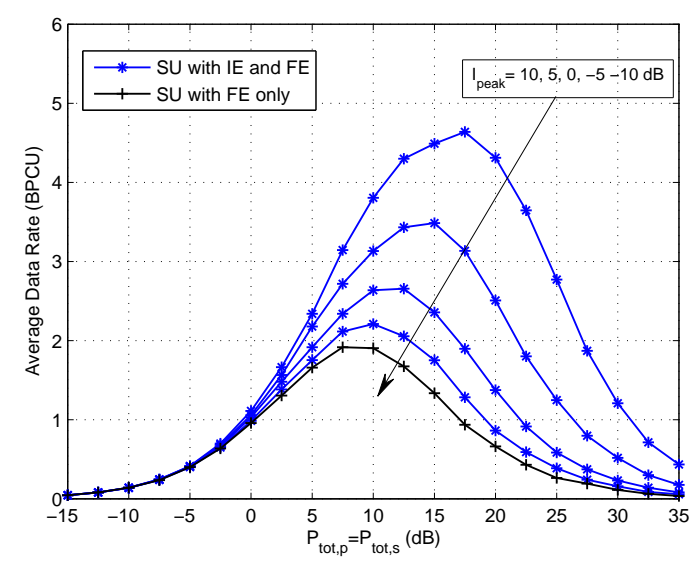

(b) Imperfect SIC.

Figure 2: Rate of the CR user with fixed $I_{\text {peak }}$ vs. $P_{t o t}=P_{t o t, p}=P_{t o t, s}$ with $N=4$.

with fixed $I_{\text {peak }}$, i.e., the SU rate given by (23) with $\alpha=0$. We notice that the rate presents again a maximum before decreasing, in this case, to zero for high SNR. However, within this extreme case, the rate has a remarkable enhancement (up to $3 \mathrm{BPCU}$ ) comparing to the FE PA precoding. In Fig. 3 we show the effect of multi-antenna diversity on the SU rate, with $P_{t o t, s}=20 \mathrm{~dB}$, considering perfect SIC and for different values of $I_{\text {peak }}$. We notice that the slope of the rate variation is almost linear, and the secondary rate reaches the upper limit (i.e., absence of the PU) above 10 antennas for $I_{\text {peak }}=10 \mathrm{~dB}$. Indeed, in this case and at high SNR, the SU rate converges to the case when PU is absent: the SU performs a Uniform Power Allocation (UPA), i.e., $N \log _{2}\left(1+I_{\text {peak }}\right)=N \log _{2}\left(1+\frac{P_{\text {tot }}}{N}\right)$.

In Fig.4, we analyze the variation of the perfect SIC PU and $\mathrm{SU}$ rate as a function of the estimation errors $\sigma_{s}^{2}$ and $\sigma_{p}^{2}$

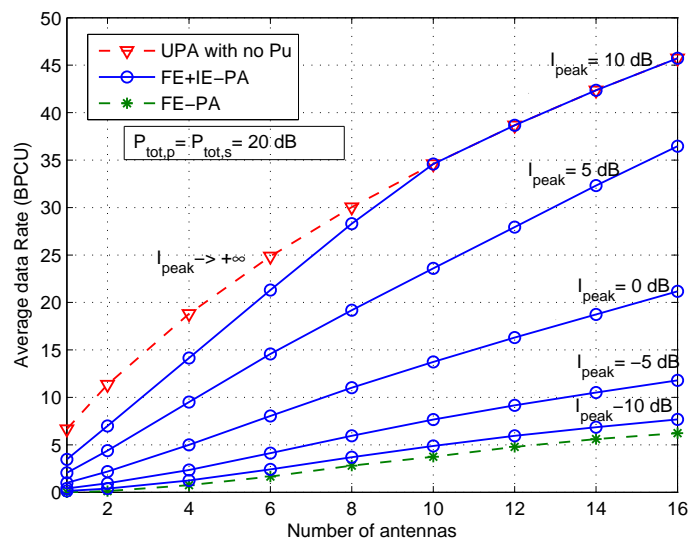

Figure 3: Rate of the CR user as a function of the number of antennas $N$ with $P_{t o t, s}=20 \mathrm{~dB}$.

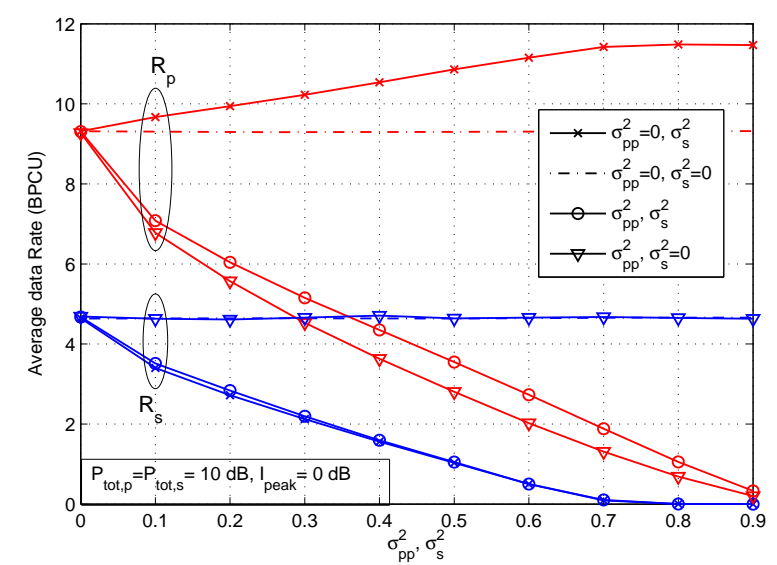

Figure 4: PU and SU rates vs. CSI estimation errors.

with $N=4, P_{t o t, p}=P_{t o t, s}=10 \mathrm{~dB}$ and $I_{\text {peak }}=0 \mathrm{~dB}$. We analyze different cases in which either one of the channels or both of them are imperfectly estimated (in the latter case they have the same estimation error). For the PU rate, $R_{p}$, we notice that when the PU channel is perfect, i.e., $\sigma_{p p}^{2}=0$, and the SU channel is imperfect, $R_{p}$ increases by $23 \%$ when $\sigma_{s}^{2}>0.7$. This result shows that the degradation of the SU channel increases the PU rate since the interference caused by the SU in the SIC becomes lower. From another side, when $\sigma_{p p}^{2}$ increases and $\sigma_{s}^{2}=0$, the rate $R_{p}$ decreases remarkably by $70 \%$ for $\sigma_{p p}^{2}=0.5$ and by $93 \%$ for $\sigma_{p p}^{2}=0.8$. However, when $\sigma_{s}^{2}>0$, these decreases become lower; $62 \%$ and $88 \%$ for $\sigma_{p p}^{2}=\sigma_{s}^{2}=0.5$ and $\sigma_{p p}^{2}=\sigma_{s}^{2}=0.8$, respectively. This fact shows again that the SU CSI imperfection enhances the PU rate. For the SU rate, we notice that the degradation of 
$R_{s}$ is $67 \%$ and almost $100 \%$ for $\sigma_{s}^{2}=0.5$ and $\sigma_{s}^{2}=0.8$, respectively, which shows that the SU rate is more sensitive to its channel estimation error than the PU. In addition, we see that the effect of the free eigenmodes mis-alignment is very limited since the SU rate, when the PU channel is imperfect, is very close to the perfect PU CSI.

\section{Optimized SECONdARY User Rate With PRIMARY TOLERANCE CONSTRAINT}

In Section III, we have adopted a conventional WPA technique in order to maximize the PU achievable rate. In this Section, we introduce a modified water-filling policy that depends on a parameter $\delta$ [15]. The parameter $\delta$ can be defined as the degradation tolerance of the PU performance, i.e., the maximum degradation that keeps the primary QoS in an acceptable level. Note that in the conventional WPA in (6), we took $\delta=0$. However, when $\delta>0$, the SU rate is expected to increase by controlling the PU power allocated to each antenna. In fact, $\delta$ provides more degrees of freedom to the SU as the solution of the PU WPA problem described in (III-A) is modified as follows $\forall j=1, \ldots, N$ :

$$
P_{p}(j, j)=\left\{\begin{array}{l}
{\left[\frac{1}{\mu}-\frac{1+I_{\text {peak }}}{\lambda_{j}^{2}}\right] \text { if }\left[\frac{1}{\mu}-\frac{1+I_{\text {peak }}}{\lambda_{j}^{2}}\right]>\delta} \\
0 \text { elsewhere. }
\end{array}\right.
$$

The higher the parameter $\delta$ is, the more available free eigenmodes are. Thus, the SU can exploit these additional free eigenmodes and enhance its achievable rate. However, an overuse of this parameter may cause the degradation of PU performance. Therefore, we need to introduce a rate degradation tolerance $0 \leq \tau \leq 1$ (i.e., a maximum rate degradation allowed by the PU) such that at fixed $P_{t o t}$ :

$$
R_{p}(\delta) \geq \tau R_{p}(\delta=0)
$$

where $R_{p}(\delta=0)$ is the PU rate achieved with the traditional WPA expressed in (III-A), i.e., no tolerance, with regards to the eigenmodes is allowed by the PU. The parameter $\tau$ determines the critical rate below which the primary QoS is not satisfied. This parameter introduces a trade-off between the PU and SU rate. In fact, when $\tau$ decreases, the critical rate, $R_{p}(\delta=0)$, decreases and hence more eigenmodes can be provided to the SU which increases its rate, $R_{p}$. Our aim is to find the optimal $\delta$ that maximizes the SU rate, $R_{s}(\delta)$ for fixed $\tau$, and satisfies the PU rate conditions expressed in (30). The optimization problem can be formulated as follows:

$$
\begin{aligned}
& \underset{\boldsymbol{P}_{s}, \delta}{\operatorname{maximize}} R_{s}(\delta) \\
\text { subject to } & \operatorname{Tr}\left(\boldsymbol{A}_{\boldsymbol{s}} \boldsymbol{P}_{\boldsymbol{s}}\right) \leq P_{t o t, s}, \\
& P_{s}(j, j) \leq I_{\text {peak }}, \forall j=1, \cdots, N-n . \\
& R_{p}\left(\delta, P_{p}\right) \geq \tau R_{p}(\delta=0) .
\end{aligned}
$$

For a given rate degradation tolerance $\tau$, in order to find the optimal $\delta^{*}$, we adopt a dichotomic algorithm based on the monotonicity of $R_{p}(\delta)$. As mentioned before, increasing $\delta$ gives more free eigenmodes for the $\mathrm{SU}$ which means less eigenmodes for the PU and thus the PU rate (i.e., $R_{p}$ decreases). Hence, $R_{p}(\delta)$ is a decreasing function and the

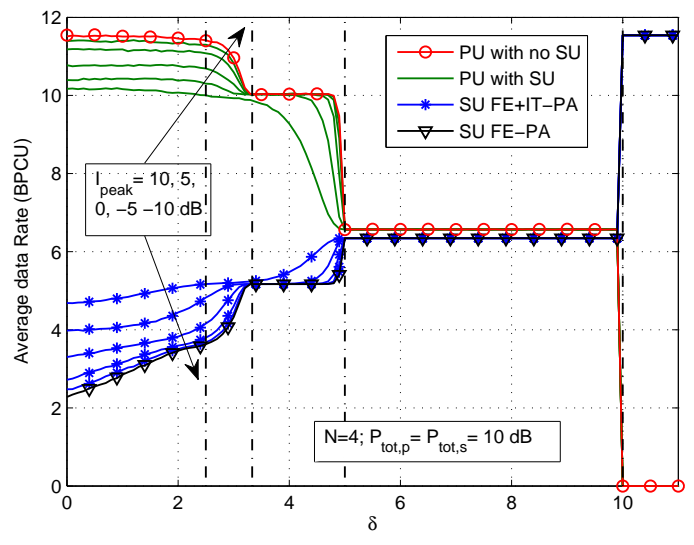

(a) Rates with $N=4$ antennas

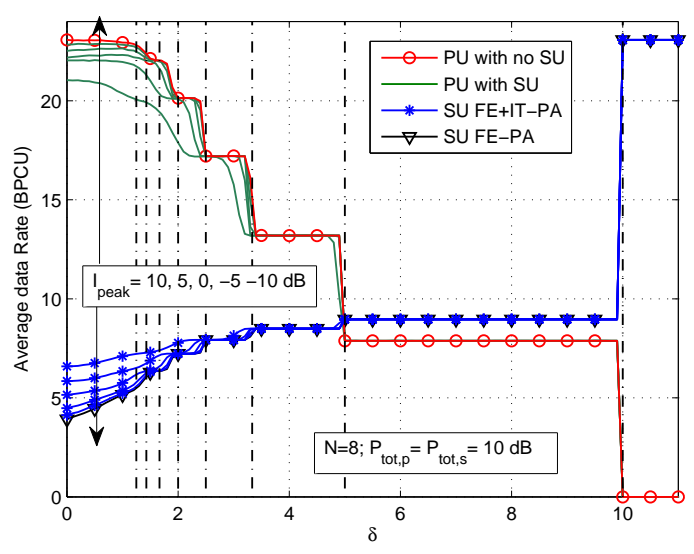

(b) Rates with $N=8$ antennas

Figure 5: Rates of the PU and the SU vs. $\delta$ for $N=4$ and $N=8$.

dichotomic algorithm is based on the monotonic decrease of $R_{p}(\delta)$. In Fig. 5.a and 5.b, we show the effect of $\delta$ on the PU and the SU rates by setting $\tau=0$ for $N=4$ antennas and $N=8$ antennas, respectively. We notice that increasing $\delta$ causes the degradation of the PU rate and the enhancement of the SU rate due to the release of more free eigenmodes to the SU. However, we notice that for $\delta<2$, the PU rate is almost constant and we have an increase in the SU rate of $10-60 \%$ depending on the values of $I_{\text {peak }}$. Meanwhile, the slope of the rates variation is different; in some intervals of $\delta$ the variation is small, e.g., for $5<\delta<10$ and $N=4$. In some values of $\delta$ the variation is large e.g., for $\delta=5,10$ for $N=4$. In fact, for a specific interval of $\delta$, there is a release of a fixed number of eigenmodes, in average, which means that the PU and SU rate remain almost the same in this interval. Consequently, the transition between two different intervals introduces the sudden rates variation. In [15], the author indicates that $\frac{P_{t o t, p}}{n+1} \leq \delta \leq \frac{P_{t o t, p}}{n}$, for $n=1, \ldots, N-1$, there is a release of $N-n$ eigenmodes that the SU can exploit. Hence, $\delta$ presents a crucial tradeoff between the PU and the SU rates. In Fig. 5, the values when $\delta=\frac{P_{t o t, p}}{n}$, for $n=1, \ldots, N-1$, are presented by a vertical dashed lines and we verify that these results hold for 


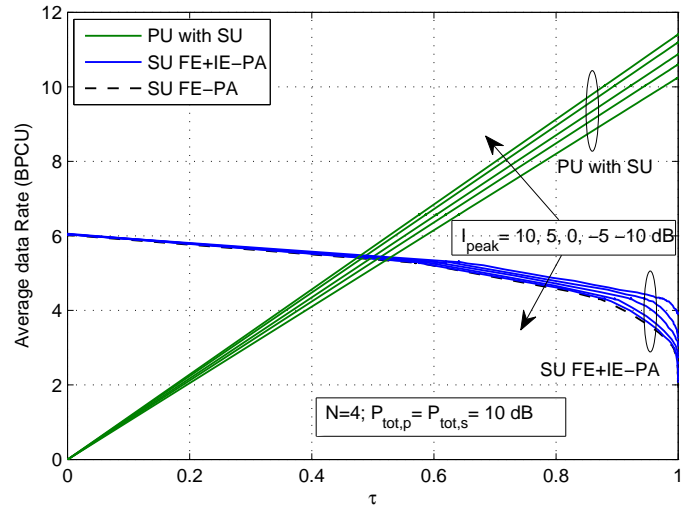

(a) Rates vs $\tau$.

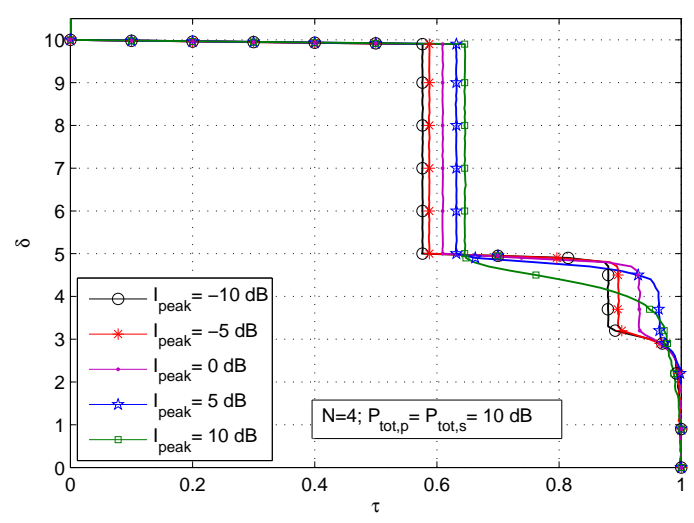

(b) $\delta$ vs $\tau$.

Figure 6: Rates of the PU and the SU vs. $\tau$ for $N=4$.

our work as well. For values of $\delta$ higher than $P_{t o t, p}$, the SU rate achieves the MIMO-WPA rate as all PU eigenmodes are free in this case.

In Fig. 6.a and 6.b, we present the PU and the SU rates and the parameter $\delta$ as function of the degradation tolerance $\tau$ for $N=4$ antennas, respectively. A first remark is that when $\tau=1$, the SU has a strict positive value which means that this gain is achieved without degrading the PU rate. However, as $\tau$ decreases toward 0 , the variation of the SU rate is relatively small (about 4 BPCU) compared to the PU rate (12 BPCU), which means that in practical cases, the values of $\tau$ should not be very low i.e., $\tau \geq 0.8$. In extreme cases, (when $\tau<0.4$ ) the SU rate overcomes the PU rate and we notice that as we increase the number of antennas $N$, the intersection between the PU and the SU rates is performed with lower values of $\tau$. In Fig. 6.b, we show how $\tau$ affects $\delta$ and therefore the number of free eigenmodes. In fact, when $\tau$ is low, i.e. the PU rate is low, we notice that the corresponding $\delta$ is at its maximum value and hence more free eigenmodes are available and SU rate increases. However. when $\tau$ is close to one the SU rate is at its minimum value which presents the trade-off between the $\mathrm{PU}$ and $\mathrm{SU}$ rate when $\tau$ is varying.

\section{Rate Region Study}

In this section, we formulate a weighted optimization problem, with perfect SIC, by constructing a single aggregate objective function that corresponds to the linear sum of the PU and SU rates expressed in (19) and (12), respectively. The sum rates are weighted with a parameter $\omega(0 \leq \omega \leq 1)$ and result in a concave function as follows:

$$
\begin{aligned}
& \underset{\boldsymbol{P}_{\boldsymbol{p}} \boldsymbol{P}_{\boldsymbol{s}}}{\operatorname{maximize}} R=\omega R_{p}+(1-\omega) R_{s} \\
& \text { subject to } \operatorname{Tr}\left(\boldsymbol{P}_{\boldsymbol{p}}\right) \leq P_{t o t, p}, \\
& \quad \operatorname{Tr}\left(\boldsymbol{A}_{\boldsymbol{s}} \boldsymbol{P}_{\boldsymbol{s}}\right) \leq P_{t o t, s}, \\
& \text { and } P_{s}(j, j) \leq I_{\text {peak }}, \forall j=1, \cdots, N-n .
\end{aligned}
$$

This optimization problem can be also solved using the Lagrange method and the resulting power profile is, $\forall j=1, \ldots, N$ :

$$
\begin{aligned}
& P_{p}{ }^{*}(j, j)=\left\{\begin{array}{c}
{\left[\frac{\omega}{\mu}-\frac{1+I_{\text {peak }}}{\lambda_{j}^{2}}\right]^{+}, \text {if }\left[\frac{\omega}{\mu}-\frac{1+I_{\text {peak }}}{\lambda_{j}{ }^{2}}\right]^{+} \geq \delta,} \\
0 \text { elsewhere, }
\end{array}\right. \\
& P_{s}^{*}(j, j)= \\
& \left\{\begin{array}{c}
\min \left\{\left[\frac{1}{A_{s}(j, j)} \frac{1-\omega}{\mu}-1\right]^{+}, I_{\text {peak }}\right\}, \forall j=1, \cdots, N-n, \\
{\left[\frac{1}{A_{s}(j, j)} \frac{1-\omega}{\mu}-1\right]^{+}, \forall j=N-n+1, \cdots, N,}
\end{array}\right.
\end{aligned}
$$

Note that when $\omega \rightarrow 0$, the optimization problem aims to maximize the SU rate only while when $\omega \rightarrow 1$ the PU rate is maximized. Other values of $\omega$ constitute a trade-off between these two extremes. In Fig. 7, we present the achievable rate

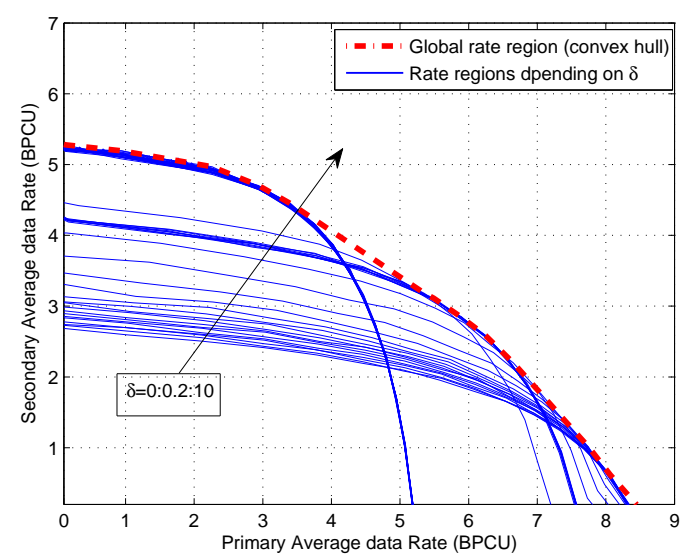

Figure 7: Rate region with various values of $\delta$.

region of the PU and the SU, with perfect SIC and various values of $\delta$ respectively with a $P_{t o t, p}=P_{t o t, s}=10 \mathrm{~dB}$. In Fig. 7.a, $\delta$ varies from 0 to 10 with a step equal to 0.2 . We notice that as $\delta$ increases, the secondary rate increases and the primary rate decreases slightly, this variation of $\delta$ gives a set of clustered rate regions related to the constant rates observed in Fig. 5 and Fig. 6, i.e., $\delta \in] 5,10[$. The upper envelop obtained as the hull convex of all the curves, plotted dashed curve, is 
considered as a global rate region involving the different values of $\delta$. Note that each point of the boundary of this envelop is achievable in the sense that it corresponds to a specific $\delta$, say $\delta_{0}$, using the power profile given in (33) and (34) along with SIC at the receiver that grantees the achievability.

\section{CONCLUSION}

In this paper, we have studied the achievable rate of the secondary cognitive user in a spectrum sharing MIMO uplink communication using a special precoding scheme. The secondary user exploits the unused eigenmodes of the primary user and shares the used ones by respecting both total power and interference temperature constraints. We showed that the secondary achievable rate increases significantly when the secondary user exploits the free eigenmodes and shares the used eigenmodes "responsibly". The impact of an imperfect CSI estimation on the primary and the secondary rates has also been highlighted. In addition, we presented a protocol of primary communication with a modified water-filling policy that achieves a fraction of the primary rate and we highlighted the gain in terms of the secondary rate. Finally, we have studied the rate region of our system and we characterized the trade-off between the primary and secondary rates.

\section{APPENDIX : ProOF OF THE THEOREM}

We start by solving two independent subproblems with the objective function (12) and one of the constraints each, similar to the theorem in [29]. Consequently the optimal power is given by the intersection of the two sub-solutions; the first is the usual water-filling scheme such as (6) and the second is the upper bound of the feasible space which is $I_{\text {peak }}$ for $j=$ $1, \cdots, N-n$. Finally, the complete optimal power expression is given by

$$
\begin{aligned}
g(\mu)= & \sum_{j=1}^{N-n} \log _{2}\left(1+\min \left\{\left[\frac{1}{A_{s}(j, j)} \frac{1}{\mu}-1\right]^{+}, I_{\text {peak }}\right\}\right) \\
& +\sum_{j=N-n+1}^{N} \log _{2}\left(1+\left[\frac{1}{A_{s}(j, j)} \frac{1}{\mu}-1\right]^{+}\right) \\
& +\mu\left(\sum_{j=1}^{N-n}\left(\min \left\{\left[\frac{1}{A_{s}(j, j)} \frac{1}{\mu}-1\right]^{+}, I_{\text {peak }}\right\}\right)\right. \\
& \left.+\sum_{j=N-n+1}^{N}\left(\left[\frac{1}{A_{s}(j, j)} \frac{1}{\mu}-1\right]^{+}\right)-P_{\text {tot }, s}\right) \cdot
\end{aligned}
$$

Now, in order to find $\mu$ we use the strong duality of our problem since our primal problem is convex [27]. Let $g(\mu)$ to be the dual function given by (35). The strong duality imposes that $g(\mu)$ is a concave, and that there exists a unique $\mu^{*}$ that minimizes $g$ and, hence, maximizes the primal function as well. Therefore, given $P_{t o t, s}$, we compute $\mu^{*}=\operatorname{argmin}_{\mu} g(\mu)$ then the optimal power $P_{s}^{*}$ and finally we compute the capacity $R_{s}^{(1)}$.

\section{REFERENCES}

[1] "Spectrum policy task force," Federal Communications Commission, Tech. Rep. ET Docket no. 02-135, Nov. 2002.

[2] J. Mitola and G. Q. Maguire, "Cognitive radio: Making software radios more personal," IEEE Personal Communications Magazine, vol. 6, no. 4, pp. 13-18, Aug. 1999.

[3] A. Goldsmith, S. Jafar, I. Maric, and S. Srinivasa, "Breaking spectrum gridlock with cognitive radios: An information theoretic perspective," Proceedings of the IEEE, vol. 97, no. 5, pp. 894-914, May 2009.

[4] J. Mitola, "Cognitive Radio: An Integrated Agent Architecture for Software Defined Radio," Ph.D. dissertation, Royal Institute of Technology (KTH), Stockholm, Sweden, May 2000.

[5] A. Ghasemi and E. S. Sousa, "Fundamental limits of spectrum-sharing in fading environments," IEEE Transactions on Wireless Communications, vol. 6, no. 2, pp. 649-658, Feb. 2007.

[6] X. Kang, Y.-C. Liang, A. Nallanathan, H. Garg, and R. Zhang, "Optimal power allocation for fading channels in cognitive radio networks: Ergodic capacity and outage capacity," IEEE Transactions on Wireless Communications, vol. 8, no. 2, pp. 940-950, Feb. 2009.

[7] S. Srinivasa and S. Jafar, "Cognitive radios for dynamic spectrum access - The throughput potential of cognitive radio: A theoretical perspective," IEEE Communications Magazine, vol. 45, no. 5, pp. 73-79, May 2007.

[8] T. Yucek and H. Arslan, "A survey of spectrum sensing algorithms for cognitive radio applications," IEEE Communications Surveys Tutorials, vol. 11, no. 1, pp. 116-130, First 2009.

[9] G. Scutari, D. Palomar, and S. Barbarossa, "Cognitive MIMO radio," IEEE Signal Processing Magazine, vol. 25, no. 6, pp. 46-59, Nov. 2008.

[10] S. Hua, H. Liu, M. Wu, and S. S. Panwar, "Exploiting MIMO antennas in cooperative cognitive radio networks," in Proc. of the IEEE INFOCOM'11, Apr. 2011, Shanghai, China, pp. 2714-2722.

[11] S. Perlaza, M. Debbah, S. Lasaulce, and J.-M. Chaufray, "Opportunistic interference alignment in MIMO interference channels," in Proc. of the 19th IEEE International Symposium on Personal, Indoor and Mobile Radio Communications (PIMRC'08), Cannes, France, Sep. 2008.

[12] X. Kang, Y.-C. Liang, and A. Nallanathan, "Optimal power allocation for fading channels in cognitive radio networks under transmit and interference power constraints," in Proc. of the IEEE International Conference on Communications (ICC'08), Beijing, China, May 2008, pp. 3568-3572.

[13] R. Zhang and Y.-C. Liang, "Exploiting multi-antennas for opportunistic spectrum sharing in cognitive radio networks," in Proc. of the IEEE 18th International Symposium on Personal, Indoor and Mobile Radio Communications (PIMRC 2007), Athens, Greece, Sep. 2007.

[14] S. Perlaza, N. Fawaz, S. Lasaulce, and M. Debbah, "From spectrum pooling to space pooling: Opportunistic interference alignment in MIMO cognitive networks," IEEE Transactions on Signal Processing, vol. 58, no. 7, pp. 3728-3741, July 2010.

[15] I. Krikidis, "Space Alignment for Cognitive Transmission in MIMO Uplink Channels," EURASIP Journal on Wireless Communications and Networking, Nov. 2010.

[16] L. Sboui, Z. Rezki, and M.-S. Alouini, "A unified framework for the ergodic capacity of spectrum sharing cognitive radio systems," IEEE Transactions on Wireless Communications, vol. 12, no. 2, pp. 877-887, Feb. 2013.

[17] I. Krikidis, "A SVD-based location coding for cognitive radio in MIMO uplink channels," IEEE Communications Letters, vol. 14, no. 10, pp. 912-914, Oct. 2010

[18] D. Hamza and S. Aissa, "An optimal probabilistic multiple-access scheme for cognitive radios," IEEE Transactions on Vehicular Technology, vol. 61, no. 7, pp. 3002-3014, Sept. 2012.

[19] J. Yang and S. Ulukus, "Delay-minimal transmission for average power constrained multi-access communications," IEEE Transactions on Wireless Communications, vol. 9, no. 9, pp. 2754-2767, Sept. 2010.

[20] L. Sboui, H. Ghazzai, Z. Rezki, and M.-S. Alouini, "On the throughput of a relay-assisted cognitive radio MIMO channel with space alignment," in 12th International Symposium and Workshops on Modeling and Optimization in Mobile, Ad Hoc and Wireless Networks (WiOpt '14), Hammamet, Tunisia, May 2014.

[21] L. Sboui, H. Ghazzai, Z. Rezki, and M. Alouini, "Achievable rate of a cognitive MIMO multiple access channel with multi-secondary users," IEEE Communications Letters, vol. PP, no. 99, pp. 1-1, Jan. 2015.

[22] "Extending LTE Advanced to unlicensed spectrum," Qualcomm Incorporated, Tech. Rep., Dec. 2013.

[23] P. Popovski, H. Yomo, K. Nishimori, R. Di Taranto, and R. Prasad, "Opportunistic interference cancellation in cognitive radio systems," in Proc. of the 2nd IEEE International Symposium on New Frontiers in 
Dynamic Spectrum Access Networks (DySPAN'07), Dublin, Ireland, Apr. 2007, pp. 472-475.

[24] S. Haykin, "Cognitive radio: Brain-empowered wireless communications," IEEE Journal on Selected Areas in Communications, vol. 23, no. 2, pp. 201-220, Feb. 2005.

[25] E. Telatar, "Capacity of multi-antenna Gaussian channels," European Transactions on Telecommunications, vol. 10, no. 6, pp. 585-595, Nov.-Dec. 1999. [Online]. Available: http://dx.doi.org/10.1002/ett.4460100604

[26] J. Blomer and N. Jindal, "Transmission capacity of wireless ad hoc networks: Successive interference cancellation vs. joint detection," in Proc. of the IEEE International Conference on Communications (ICC '09), Dresden, Germany, June 2009, pp. 1-5.

[27] S. Boyd and L. Vandenberghe, Convex Optimization. Cambridge University Press, 2004.

[28] A. Tall, Z. Rezki, and M.-S. Alouini, "MIMO channel capacity with full CSI at low SNR," IEEE Wireless Communications Letters, vol. 1, no. 5, pp. 488-491, Oct. 2012.

[29] L. Sboui, H. Ghazzai, Z. Rezki, and M.-S. Alouini, "Achievable rate of cognitive radio spectrum sharing MIMO channel with space alignment and interference temperature precoding," in Proc. of the IEEE International Conference on Communications (ICC'13), Budapest, Hungary, June 2013, pp. 2656-2660.

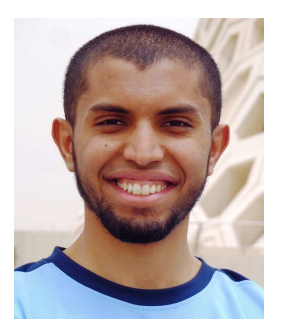

Lokman Sboui (S'11) was born in Cairo, Egypt. He received the Diplome dIngénieur degree with honors from Ecole Polytechnique de Tunisie (EPT), La Marsa, Tunisia, in 2011, the MS degree from King Abdullah University of Science and Technology (KAUST) in May 2013. He is currently a Ph.D. candidate in the Electrical Engineering program of KAUST. His current research interests include: performance of cognitive radio systems, low SNR communication, MIMO communication, Relaying performances, energy efficient power allocation and

green wireless sensor networks.

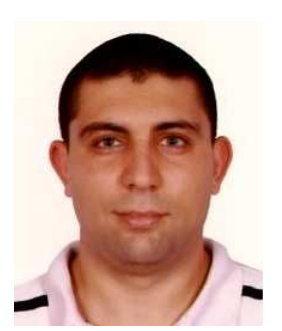

Hakim Ghazzai (S'12, M'15) was born in Tunisia. $\mathrm{He}$ is currently working as a research scientist at Qatar Mobility Innovations Center (QMIC), Doha, Qatar. He received his $\mathrm{PhD}$ degree in Electrical Engineering from King Abdullah University of Science and Technology (KAUST) in Saudi Arabia. $\mathrm{He}$ received his Diplome d'Ingenieur in telecommunication engineering with highest distinction from the Ecole Superieure des Communications de Tunis (SUP'COM), Tunis, Tunisia in 2010 and his Master degree in High-Rate Transmission Systems from the same institute in 2011. His general research interests are at the intersection of wireless networks, green communications and optimization.

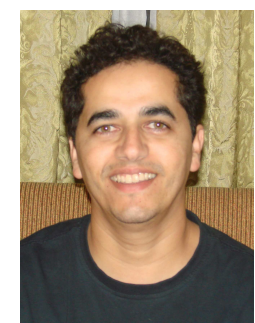

Zouheir Rezki (S'01, M'08) was born in Casablanca, Morocco. He received the Diplome d'Ingénieur degree from the École Nationale de l'Industrie Minérale (ENIM), Rabat, Morocco, in 1994, the M.Eng. degree from École de Technologie Supérieure, Montreal, Québec, Canada, in 2003, and the Ph.D. degree from École Polytechnique, Montreal, Québec, in 2008, all in electrical engineering. From October 2008 to September 2009, he was a postdoctoral research fellow with Data Communications Group, Department of Electrical and Computer Engineering, University of British Columbia. He is now a research scientist at King Abdullah University of Science and Technology (KAUST), Thuwal, Makkah Province, Saudi Arabia. His research interests include: performance limits of communication systems, cognitive and sensor networks, physicallayer security, and low-complexity detection algorithms.

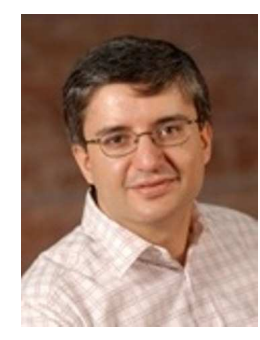

Mohamed-Slim Alouini (S'94, M'98, SM'03, F'09) was born in Tunis, Tunisia. He received the Ph.D. degree in Electrical Engineering from the California Institute of Technology (Caltech), Pasadena, CA, USA, in 1998. He served as a faculty member in the University of Minnesota, Minneapolis, MN, USA, then in the Texas A\&M University at Qatar, Education City, Doha, Qatar before joining King Abdullah University of Science and Technology (KAUST), Thuwal, Makkah Province, Saudi Arabia as a Professor of Electrical Engineering in 2009. His current research interests include the modeling, design, and performance analysis of wireless communication systems. 